\title{
Zur Geschichte des Bronchialkarzinoms
}

\section{G. Dhom}

\section{The History of Bronchial Carcinoma}

Es gibt wohl keinen zweiten bösartigen Tumor, dessen Geschichte eine ähnlich dramatische Entwicklung genommen hat, wie die des Lungen- und Bronchialkarzinoms. Noch in der Mitte des 19. Jahrhunderts war ein primäres Bronchialkarzinom unbekannt, die Abgrenzung eines primären Lungenkarzinoms von Lungenmetastasen unsicher.

In dieser Arbeit wird versucht, wichtige Meilensteine in der Geschichte des Lungen- und Bronchialkarzinoms vorzustellen, wobei der Schwerpunkt auf der pathologischen Anatomie liegt.

In Rokitanskys Lehrbuch der Pathologischen Anatomie von 1862 (3. Auflage) lesen wir: „An den Bronchien beobachtet man bisweilen eine vom Bronchialstamm an auf seine Verästelung fortgesetzte krebsige Entartung, wobei die Wände der Bronchien verdickt, rigid, das Lumen derselben verengt, die innere Fläche derselben höckerig uneben erscheinen. Die Entartung trifft mit Carcinom der Bronchialdrüsen, mit ausgebreitetem Carcinom der Costalpleura zusammen und geht ohne Zweifel von ihnen aus“ [1]. Mit „Bronchialdrüsen“ sind hier die bronchusnahen Lymphknoten gemeint. Obwohl also Rokitansky einprägsam den makroskopischen Befund eines Bronchialkarzinoms schildert, verlegt er doch den Ursprung des Tumors in die angrenzenden Lymphknoten.

Die epitheliale Genese der Karzinome ist zu dieser Zeit bekanntlich noch keineswegs anerkannt, ja von Virchow bestritten. Erst mit den Arbeiten von Thiersch 1865 [2] und Waldeyer 1867/72 [3] wird sich die Lehre vom epithelialen Ursprung der Karzinome durchsetzen. So verwundert es nicht, dass noch 1867 Theodor Langhans, damals Assistent am Pathologischen Institut Würzburg, in einer umfangreichen Arbeit über Krebs und Kankroid der Lunge [4] Tumoren beschreibt, die er einmal aus dem bindegewebigen Gerüst der Lunge hervorgehen lässt, zum anderen aber auch in der „Matrix“ der Alveolen sucht. Er gewinnt seine Befunde an Sammlungspräparaten. Die Beschreibung lässt zweifelsfrei erkennen, dass es sich bei seinen Beobachtungen um Metastasenlungen handelt.

1871 veröffentlicht Langhans dann aus Marburg den ersten gesicherten Fall eines primären Bronchialkarzinoms [5]. Der 40-jährige Mann leidet seit einem Jahr an Atembeschwerden mit wiederholten Erstickungsanfällen. Bei der Autopsie findet man eine Stenose des rechten Hauptbronchus (Abb.1). Es handelt sich um ein Plattenepithelkarzinom, das Langhans von den Schleimdrüsen der Bronchialschleimhaut ausgehen lässt. Bei der Deutung der epithelialen Genese seines Tumors bezieht er sich jetzt ausdrücklich auf Waldeyers Befunde an Mammakarzinomen. 1872 veröffentlicht M. Perls aus Königsberg den 2. Fall eines metastasierenden Bronchialkarzinoms mit plattenepithelialem Bau [6]. Die Histogenese dieses Geschwulstleidens ist Perls jedoch keineswegs klar, lässt er doch die Lebermetastasen aus den benachbarten Leberzellen hervorgehen und spricht sich „entschieden gegen die Alleinherrschaft der Implantationstheorie aus, wie Waldeyer sie lehrt" (pag. 462).

In den nachfolgenden Jahren mehren sich jedoch autoptische Berichte primärer Lungen- und Bronchialkarzinome. Lapidar formuliert hierzu Rindfleisch 1873 in seinem Lehrbuch der Pathologischen Gewebelehre [7] jedoch: „Die Lunge ist zu primärer Geschwulstbildung ausserordentlich wenig geneigt“. Dabei ist es bemerkenswert, dass für Rindfleisch „ausschließlich das Stroma der Lunge“ für die Genese in Betracht kommt. Vom Bronchialkrebs ist bei Rindfleisch noch keine Rede. Im damals tonange-

Rubrikherausgeber: R. Kropp (federführend), U. Costabel, H. S. Fuchs, C. Habrich, H. Jungbluth, H. J. Klippe, N. Konietzko, R. Loddenkemper, G. Neumann, S. Schulz, M. Teschner

Korrespondenzadresse

Prof. Dr. med. Georg Dhom · Am Webersberg 20 • 66424 Homburg/Saar

Bibliografie

Pneumologie 2004; 58: 680-685 (c) Georg Thieme Verlag KG Stuttgart · New York

DOI $10.1055 / \mathrm{s}-2004-818417$

ISSN 0934-8387 


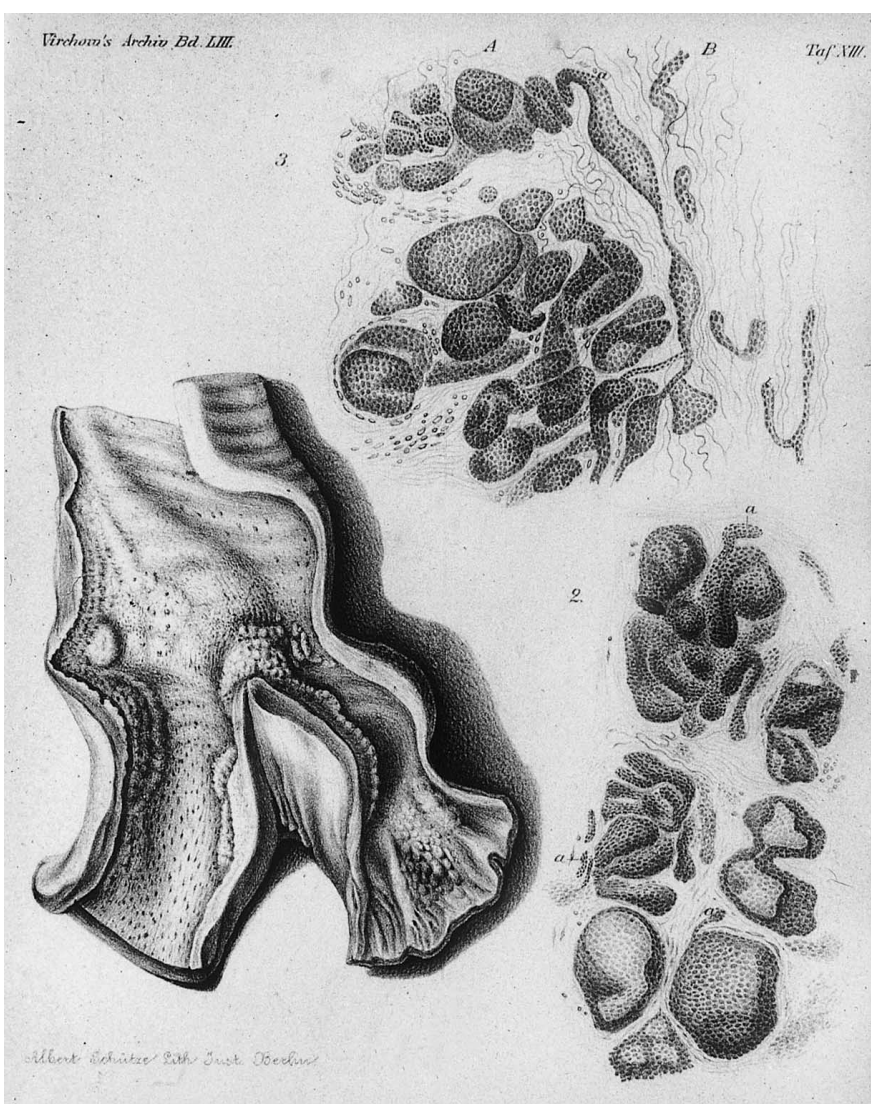

Abb. 1 Th. Langhans. Primärkrebs der Trachea und der Bronchien. Fig. 2 u. 3: „Die Endbläschen der Drüsen in mannigfacher Umbildung zu Krebszellsträngen begriffen“. Virchows Arch path Anat 1871; $53: 470-484$.

benden britischen Lehrbuch Lectures of Pathological Anatomy von S. Wilks und W. Moxon 1875, 2. Auflage, [8] wird eindeutig zwischen primären und sekundären Lungengeschwülsten unterschieden: „We would therefore say that cancer of the lung is generally of two kinds - that in which die parenchyma is filled with cancerous masses secondary to a similar affection in another part; and that in which the lung is occupied by a mass of disease, perhaps limited to the chest and which, in contradistinction to the other, may be called primary, although it has its origin really in the bronchial tissues“ (pag. 351). Freilich: Auch Wilks und Moxon müssen gestehen: „A true primary cancer of the lung is so rare that we have only one specimen to show“ (pag. 352).

Eine Zusammenstellung von 70 kasuistischen Mitteilungen primärer Lungenkarzinome verdanken wir 1896 H. Pässler aus Breslau [9]. Nur vollständige Autopsien können damals Klarheit über den primären Lungenkrebs schaffen. Unter 54 Fällen, deren Beschreibung Pässler eine Beurteilung erlaubt, sind 47 primäre Bronchialkarzinome. Pässler ist wohl auch einer der ersten Autoren, die eine mikroskopisch-zytologische Sputumuntersuchung für die Diagnose des Bronchialkarzinoms am Patienten fordern. Unter 4 eigenen Fällen findet sich ein „kleinzelliger Cylinderzellenkrebs“, ein verhornendes Plattenepithelkarzinom, ein Zylinderzellkarzinom mit Verschleimung. „Eine Form des primären Lungenkarzinoms, welche sich ausschließlich auf das Lungenparenchym mit völliger Freilassung aller großen und kleinen Bronchien beschränkt, ist nicht sicher bekannt“" (pag. 248).
Am Ende des 19. Jahrhunderts können über die Häufigkeit des Bronchialkarzinoms unter bösartigen Geschwülsten Autopsiestatistiken verlässliche Auskunft geben. In Breslau findet Pässler unter 870 Karzinomen 16 primäre Lungenkarzinome (1,83\%). Mit weitem Abstand führt der Magenkrebs mit 319 Fällen die Liste an. In Chemnitz findet Briese zwischen 1898 und 191660 Fälle von primärem Lungen- und Bronchialkrebs unter 1287 Krebsfällen, also 4,51 \% [10]. Das ist der 6. Rang unter seinen Krebsfällen. Brieses eigene Beobachtung schildert ein klassisches doppelseitiges Alveolarzellkarzinom.

Die Zunahme der Lungen- und Bronchialkarzinome in der 1. Hälfte des 20. Jahrhunderts ist deutlich an den Autopsiestatistiken aus Bern 1900 - 1939 [11], Hamburg-Eppendorf 1898 - 1923 [12], Leipzig 1920-1951 [13] und Dresden 1852-1951 [14] erkennbar. In Bern steigt der Anteil von 1,2\% auf 7,6\% aller Karzinome, in Hamburg von 1,7\% auf 9,4\%, in Leipzig von $9 \%$ auf $21 \%$. Bei den Männern nimmt in Leipzig am St. Georg Krankenhaus in den Jahren 1942 - 1951 der Lungenkrebs mit 35\% aller Krebsfälle eine Spitzenposition ein, der Magenkrebs wird an relativer Häufigkeit übertroffen. Zu Recht stellt H. Grosse in seiner 100-jährigen Lungenkrebsstatistik aus dem Pathologischen Institut DresdenFriedrichstadt fest: „Da die Gesamtkrebshäufigkeit bei den Männern im Laufe der 100 Jahre um das 3,3fache, die Lungenkrebshäufigkeit aber um das 18 fache zunimmt, darf die Lungenkrebszunahme als eine echte, nicht allein durch Überalterung bedingte Zunahme angesehen werden“ (pag. 332).

Die Autopsiestatistiken sagen natürlich nichts über bevölkerungsbezogene Inzidenzen aus. Die erhebliche Zunahme des Lungen- und Bronchialkarzinoms haben die nach dem 2. Weltkrieg eingerichteten epidemiologischen Krebsregister weltweit offengelegt [15]. In den letzten 20 Jahren beobachtet man eine divergierende Entwicklung bei Männern und Frauen, wie am Beispiel der Bevölkerung des Saarlandes gezeigt werden kann (Abb. 2 und Abb. 3) [16]. Bei den Männern ist seit 1970 kein Anstieg, eher ein Trend zur Reduktion der Inzidenz und Mortalität zu beobachten. Bei den Frauen kommt es dagegen fast zu einer Verdreifachung der Werte [17]. Der weibliche Lungenkrebs macht 1997 im Saarland 4,8\% aller Krebserkrankungen bei Frauen aus und ist nun etwa gleich häufig wie der Mastdarmkrebs bei der Frau. Mit 17,5\% nimmt der Lungenkrebs bei den Männern immer noch eine Spitzenposition ein, jetzt dicht gefolgt vom Prostatakrebs.

\section{Rauchen als ätiologischer Faktor}

Die fast explosive Zunahme des Lungenkrebses seit Anfang des 20. Jahrhunderts hat alle Forscher herausgefordert, den Ursachen nachzugehen. Zunächst wurden im Gefolge der Grippeepidemie 1918 Plattenepithelmetaplasien der Bronchialschleimhaut gesehen und von Siegmund [18] als Präkanzerosen beschrieben. Auch Berblinger [19] weist auf die Zunahme der Lungenkrebse nach der Grippeepidemie und auf die Bedeutung der Influenza als mögliche Krebsursache hin. Eine der ersten Anmerkungen zum Thema Rauchen und Lungenkrebs stammt 1923 von Theodor Fahr, Hamburg. In einer Diskussionsbemerkung auf der 19. Tagung der Deutschen Pathologischen Gesellschaft in Göttingen zu einem Vortrag von Teutschländer über „Metaplasie und 

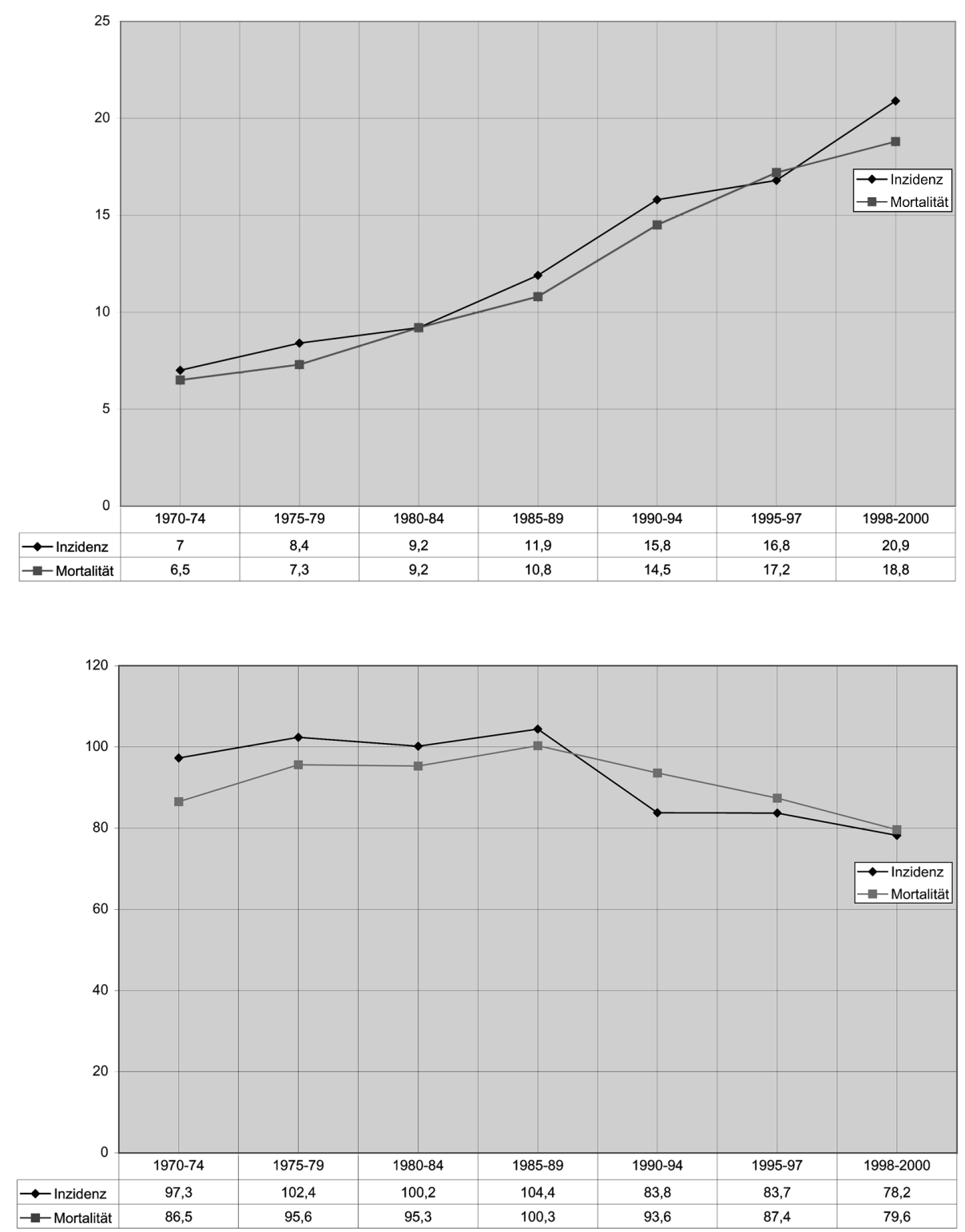

Abb. 2 Saarländisches Krebsregister. Inzidenz und Mortalität des Bronchialkarzinoms (ICD 162) (Europa-Standard) 1970-2000, Frauen.

Abb. 3 Saarländisches Krebsregister. Inzidenz und Mortalität des Bronchialkarzinoms (ICD 162) (Europa-Standard) 1970-2000, Männer.
Krebsbildung“ bemerkt er: „Als Reiz für die Entstehung des Bronchialkrebses kommt m.E. nur eine chronisch wirkende Schädlichkeit in Betracht, schwerlich eine Vergiftung mit Kampfgasen, viel eher das Inhalieren beim Zigarettenrauchen, welches zweifellos zugenommen hat“ [20].

Eine frühe zusammenfassende Übersicht über „Tabak und Tabakrauch als ätiologischer Faktor des Bronchialcarcinoms“ wird 1930 von Lickint, Chemnitz, publiziert [21]. Zu dieser Zeit ist schon lange bekannt, dass man experimentell mit Tabakteerpinselungen Epithelproliferationen an der Haut von Ratten, Mäusen und Kaninchen erzeugen kann. Auch sind die Zusammenhänge zwischen Lippen-, Mundschleimhaut- und Zungenkrebs mit dem Rauchen bekannt. Lickint weist nicht nur auf „die erschreckende Zunahme des Zigarettenrauchens“ hin, er sieht ein Risiko auch im Passivrauchen: „Es gelangen also tagtäglich sowohl bei Rauchern als aber auch in nicht zu unterschätzendem Maße beim Nichtraucher durch Aufenthalt in rauchigen Räumen Tabakverbrennungsprodukte an das Epithel der Luftwege“. In einer zweiten Arbeit veröffentlicht Lickint 1935 selbst erhobene Rau- cheranamnesen bei Patienten mit Bronchialkrebs [22]. Von 36 Männern sind 34 starke Raucher.

1937 berichtet A. H. Roffo, Buenos Aires, von seinen Erfahrungen mit „Tabak als krebserzeugendes Agens“ in der sog. Rauchstraße: Lippe - Zunge - Larynx - Gaumensegel - Pharynx. Unter 5000 krebskranken Frauen finden sich nur 42 Fälle von Krebs in der sog. Rauchstraße, aber alle sind starke Raucherinnen. Experimentell belegt er, dass es die Kohlenwasserstoffe im Tabakteer seien, die kanzerogen sind [23].

1940 veröffentlicht H.F. Müller Zahlen des Statistischen Reichsamtes über den Tabakkonsum in Deutschland [24]. Der Verbrauch an Zigaretten ist seit 1907 um das 5fache gestiegen. Müller führt daraufhin systematische Raucheranamnesen bei den Patienten der Kölner Universitäts-Poliklinik ein und erforscht diese auch mit Fragebogen bei den Angehörigen verstorbener Bronchialkarzinom-Patienten. Gegenüber einer Vergleichsgruppe findet er bei 96 Krankheitsfällen ein starkes Überwiegen extremer und starker Raucher [24]. 
Der letzte und schlüssige Beweis von der überragenden Rolle des Rauchens in der Ätiologie des Bronchialkarzinoms wird nach dem 2. Weltkrieg von der analytischen Epidemiologie erbracht. An 684 Fällen belegen 1950 E. L. Wynder und E. A. Graham, St. Louis, dass $96 \%$ der Patienten mit Lungenkrebs länger als 20 Jahre geraucht haben. Über die Hälfte dieser Patienten waren exzessive oder Kettenraucher. In einer Kontrollgruppe waren nur 19,1 \% vergleichbar starke Raucher. $2 \%$ der männlichen Patienten sind Nichtraucher [25].

Seit dieser ersten, umfassenden epidemiologischen Studie gibt es eine kaum mehr überschaubare Zahl retro- und prospektiver Studien aus zahlreichen Ländern der Welt, die das Zigarettenrauchen an die erste Stelle der Ursachen des Bronchialkarzinoms stellen. Gemeinhin wird die kanzerogene Wirkung des Tabakrauchs auf 3,4 Benzpyren bezogen. H. Druckrey und R. Preussmann haben aber schon 1962 auf die im Tabakrauch nachweisbaren stark kanzerogenen Nitrosamine hingewiesen [26].

\section{Uranbergbau und Lungenkrebs}

Unter den beruflichen Risiken spielt in Deutschland der sog. Schneeberger Lungenkrebs eine bedeutende Rolle. Die „Bergsucht" der Bergleute in den schon seit 1168 betriebenen Silberminen im Erzgebirge war bereits Paracelsus bekannt. 1879 erkennen Härting und Hesse, dass es sich bei dieser Bergkrankheit um Lungenkrebs handelt [27]. 1926 wird der Schneeberger Lungenkrebs, seine Klinik und Pathologie von Rostoski, Saupe und Schmorl dargestellt [28]. Von 154 untersuchten Bergleuten sind im Laufe der Beobachtungszeit von 31/2 Jahren 21 gestorben, davon 13 an einem Lungenkarzinom. Histologisch findet Schmorl plattenepitheliale, undifferenzierte und kleinzellige Typen, wobei ihm häufige Mischformen auffallen. Die Ätiologie des Schneeberger Lungenkrebses ist zu dieser Zeit noch unbekannt. Man vermutet, dass der eingeatmete Staub entweder besonders gefährliche Eigenschaften aufweist, oder es müssen konkurriende Momente hinzutreten. Rostoski und Saupe denken dabei auch an eine „aktinisch bedingte Reizwirkung“, liegt doch der Radiumgehalt des Staubes in Gesteinsproben über dem „als normal zu betrachtenden Grad“ (pag. 373).

Das Drama des Uranbergbaus im Erzgebirge wird in Deutschland erst nach der Wiedervereinigung in seinem ganzen Umfang offengelegt. Bis dahin war das Geschehen von der Betreibergesellschaft, der „Wismut AG“, einer strengen Geheimhaltung unterworfen. Piekarski und Morfeld haben 1997 die Geschichte dieses Dramas eindrucksvoll dargelegt [29]. Der Hauptverband der gewerblichen Berufsgenossenschaften konnte jetzt eine „Zentrale Erfassungs- und Betreuungsstelle Wismut" einrichten. Hier werden sowohl die alten Daten ausgewertet, als auch alle neuen Befunde bei den noch lebenden ehemaligen Bergleuten gesammelt. Die aktuellen Schätzungen liegen z.Zt. bei etwa 11000 Lungenkrebsfällen bei 400000 Bergleuten unter Risiko. Die bisher größte prospektive Kohorten-Studie an über 58000 Uranbergarbeitern der früheren Wismut AG wird 2002 von Kreuzer und Mitarbeitern vorgelegt [30]. Neue histologische Befunde an 240 Lungenkarzinomen, die zwischen 1991 und 1995 beobachtet wurden, zeigen, dass der dominierende Typ mit $43 \%$ das Plattenepi- thelkarzinom ist, gefolgt vom Adenokarzinom (26\%) und dem kleinzelligen Karzinom mit 23\%. $8 \%$ sind sonstige Typen [31].

Nachdem 1997 die Quarzstaubbelastung von der International Agency for Research on Cancer (IARC) als karzinogen eingestuft wurde, ergab sich für den Bergbau die Frage, ob die Bergleute im Steinkohlenbergbau einem erhöhten Krebsrisiko ausgesetzt sind. Für den deutschen Steinkohlenbergbau wird dies an einer Kohorte von 4632 Bergleuten des saarländischen Steinkohlenbergbaus prospektiv geprüft [32]. Die bisherigen Ergebnisse der Mortalitätsstudie haben keine erhöhte Gesamt- oder Krebsmortalität (SMR 0,80) ergeben. Auch besteht keine erhöhte Lungenkrebsmortalität (SMR 0,79). In enger Zusammenarbeit mit dem Saarländischen Krebsregister werden die Inzidenzraten der noch lebenden Bergleute weiter verfolgt [32].

\section{Asbest und Krebsrisilko}

Unter den beruflichen Risiken spielt heute Asbest die überragende Rolle. Im Jahr 2000 verzeichnet der Hauptverband der Gewerblichen Berufsgenossenschaften in Deutschland 957 Todesfälle durch Asbest, hier etwa zur Hälfte Folge eines Mesothelioms oder eines Lungenkrebses [33]. Zwischen 1993 und 2002 schwankt die Zahl anerkannter Asbest-bedingter Lungen- und Kehlkopfkrebse (BK 4104) zwischen 436 und 786. Auf einer Konferenz der New York Academy of Sciences: „Biological Effects of Asbestos“ 1965 wird Asbest als das „Mineral des 20. Jahrhunderts" bezeichnet, dessen Anwendung in 60 Jahren um das 1000fache gestiegen sei [34]. Die ersten Beobachtungen von Lungenkarzinomen bei Asbestose stammen aus den 30er-Jahren. 1938 fordert M. Nordmann, Hannover, mit 2 eigenen Beobachtungen die Anerkennung eines Berufskrebses beim Asbestarbeiter [35]. Ein Zentrum der Asbestindustrie in Deutschland war Dresden. Auf der New Yorker Konferenz berichten Jacob und Anspach über ihre Erfahrungen an 2636 Asbestarbeitern der Dresdner Industrie - Männer und Frauen - aus den Jahren 1952 - 1964 [36]. Sie beobachten 721 Fälle von Asbestose mit 22 Lungenkarzinomen und 6 Pleuramesotheliomen. Charakteristisch ist eine im Mittel 30 Jahre währende Latenzzeit zwischen dem Beginn der Exposition und der Krebserkrankung. Bekannt ist die exponenzielle Steigerung des Krebsrisikos durch Rauchen. Nach Selikoff u. Mitarb. ist das Risiko des rauchenden Asbestarbeiters gegenüber dem Nichtraucher ohne Asbestexposition auf das 92fache erhöht [37].

Der Hauptverband der Gewerblichen Berufsgenossenschaften erwartet das Maximum Asbest-bedingter Erkrankungen in Deutschland erst zwischen 2005 und 2015.

\section{Histologische Klassifikation}

Die histologische Klassifikation der Lungen- und Bronchialkarzinome folgt heute den Empfehlungen der WHO, die dafür bereits 1958 ein Referenzzentrum etabliert hat [38]. Die Nomenklatur wird durch die morphologische Vielfalt kompliziert. Bereits 1931 hatte W. Fischer darauf hingewiesen, dass im gleichen Tumor wechselnde histologische Befunde gefunden werden können [39]. Die Häufigkeitsverteilung der einzelnen Typen ist in ei- 
nem unausgelesenen autoptischen Beobachtungsgut anders als in operativ gewonnenen Lungen. 1954 gibt G. Kahlau [40] aus dem laufenden Frankfurter Obduktionsgut mit 190 Lungenkrebsfällen der Jahre 1949-1952 folgende Verteilung an:

1. Plattenepithelkrebse $36,9 \%$

2. Kleinzellige Krebse $46,3 \%$

3. Polymorphzellige Krebse $7,4 \%$

4. Atypische Krebse 3,2\%

5. Zylinderzellkrebse $6,8 \%$

Lüdecke hat schon 1953 gezeigt, dass im Operationsgut der Chirurgischen Universitätsklinik München unter 125 Bronchialkarzinomen der Plattenepithelkrebs mit $70,4 \%$ bei weitem überwiegt, was zweifellos mit der oft günstigeren Topografie und dem langsameren Wachstum dieses Typs im Vergleich zu den undifferenzierten, vor allem kleinzelligen Formen zusammenhängt [41].

Die Veränderungen des Bronchialepithels in ihrer Abhängigkeit vom Rauchen wurde 1957 von O. Auerbach und seiner Gruppe ausführlich dargestellt [42]. Die Häufigkeit abnormer Epithelbefunde steigt mit steigendem Tabakkonsum.

Auf die Basalzellwucherungen und Plattenepithelmetaplasien hatte schon 1924 Krompecher aufmerksam gemacht. Er sieht in den Basalzellwucherungen präneoplastische Veränderungen und will sie Dysplasie nennen [43].

Die Geschichte des Sonderfalles „kleinzelliges Bronchialkarzinom" beginnt mit der Abgrenzung dieses Tumors von Sarkomen, speziell des Mediastinums. Die kleinen, undifferenzierten Zellen werden als haferkornähnlich (oat-cell carcinoma) beschrieben. 1926 betont Barnard, dass diese Tumoren Karzinome sind: „The so-called oat-cell sarcoma of the posterior mediastinum is a medullary carcinoma of bronchi“ [44]. Bei Marchesani aus Innsbruck 1924 heißen diese Tumoren noch Basalzellenkrebse. Er leitet sie richtig von den Basal- bzw. Reservezellen des Bronchialepithels ab [45]. Dass es sich dabei um einen eigenständigen Tumortyp handelt, wird ausführlich 1962 von Watson und Berg dargestellt [46]. In den Zellen lassen sich neurosekretorische Granula nachweisen [47]. Histogenetisch leitet sich der Tumor vom Helle Zellen-System der Bronchialschleimhaut ab [48,49]. Entsprechend können kleinzellige Bronchialkarzinome hormonell aktiv sein. Bereits 1958 haben Cheng u. Mitarb. eine erste Beobachtung eines Cushing-Syndroms bei Bronchialkarzinom aus China mitgeteilt [50].

1967 wird die erste internationale histologische Klassifikation der Lungentumoren der WHO von Kreyberg, Liebow und Uehlinger vorgelegt [51], der 1977 eine überarbeitete Fassung folgt [52].

Ein eigenständiger seltener Lungentumor ist das bronchiolo-alveoläre Karzinom, das vielfach einfach als Alveolarzellkarzinom bezeichnet wird. 1876 beschreibt L. Malassez die erste Beobachtung eines doppelseitigen multinodulären Alveolarzellkarzinoms [53]. Über den diffusen, einer Lobärpneumonie ähnelnden Typ berichtet $1903 \mathrm{~J}$. H. Musser [54].

Ein klassisches Beispiel eines doppelseitigen Alveolarzellkarzinoms wird 1920 von Briese aus Chemnitz mitgeteilt: „Beide Lun- gen fühlen sich fest an und sind ungemein schwer. Die Schnittflächen werden, man mag einschneiden, wo man will, von graurötlichen oder mehr grauen, durchscheinenden, vielfach geradezu glasig-schleimigen Erhebungen eingenommen. Die entscheidenden Veränderungen spielen sich im Inneren, am Alveolarepithel ab. An die Stelle der Plattenzellen sind hohe, dicht gedrängte, gegen die Lichtung hin oft überquellende, nicht flimmernde Zylinderzellen in einer Schicht getreten". Für die Ableitung dieses Neoplasmas vom Alveolarepithel spricht alles und nichts spricht dagegen, so Briese [10].

Es gehört seit je zur Definition des Alveolarzellkarzinoms, dass makroskopisch die Bronchien unbeteiligt sind und dass bei der Autopsie kein anderweitiger Primärtumor gefunden wird. Die Diagnose Alveolarzellkarzinom wird damit zum Teil eine Diagnose per exclusionem, können doch metastatische Absiedelungen in den Lungen, etwa von einem Pankreas- oder Rektumkarzinom, das Bild eines Alveolarzellkarzinoms vortäuschen, wenn die Geschwulstzellen die Alveolarwände besiedeln und wie eine Tapete auskleiden. Eck, der besonders auf diesen Metastasierungstypus aufmerksam gemacht hat, geht in seiner Schlussfolgerung aber sicher zu weit, wenn er meint, das „Alveolarzellkarzinom“ sei „nichts anderes als eine eigenartige Form der Krebsmetastasierung“" [55].

Schon die gelungene Heilung eines Alveolarzellkarzinoms nach Lob- oder Pneumonektomie spricht für die Eigenständigkeit dieses Lungenkarzinoms, wie schon ein früher Fall von Skorpil 1941 zeigt [56]. 1949 haben Delarue und E.A. Graham ein weiteres erfolgreich behandeltes Alveolarzellkarzinom publiziert [57]. Der Terminus Lungenadenomatose [58] hat sich nicht durchgesetzt. In der Klassifikation der WHO 1977 werden als Lungenadenome nur noch eindeutig gutartige epithelial-drüsige Tumoren zusammengefasst [59].

Das bronchiolo-alveoläre Karzinom ist mit 1-2\% der primären Lungen- und Bronchialkarzinome selten. Watson und Smith haben am Memorial Hospital New York 33 Fälle unter 1585-malignen Lungentumoren beobachtet, 70\% davon gehören zum nodulären und 30\% zum diffusen Typ [60]. Histogenetisch kommen sowohl Zellen des bronchiolären Epithels wie auch Pneumozyten Typ II in Betracht. Die Tumorzellen können schleimbildend sein, teilweise enthalten sie elektronenmikroskopisch nachweisbare osmiophile, lamelläre Einschlusskörperchen, wie Pneumozyten vom Typ II [61,62].

Die Prognose des Lungenkrebses (ICD 162) ist nach wie vor schlecht. In den deutschen epidemiologischen Krebsregistern der ehemaligen DDR und des Saarlandes liegen die relativen 5-Jahres-Überlebensraten um 10\% [63]. 


\section{Literatur}

${ }^{1}$ Rokitansky C. Lehrbuch der Pathologischen Anatomie. 3. Band. 3. Auflage. Wien: Braumüller, 1861: 26

${ }^{2}$ Thiersch C. Der Epithelialkrebs namentlich der Haut. Leipzig: Engelmann, 1865

${ }^{3}$ Waldeyer W. Die Entwicklung der Karzinome. Virchow Arch path Anat 1867/1872; 41/55: 477-522/67-158

${ }^{4}$ Langhans Th. Ueber Krebs und Cancroid der Lunge nebst einem Anhang ueber Corpora amylacea in der Lunge. Virchows Arch path Anat 1867; 38: 497-536

${ }^{5}$ Langhans Th. Primärer Krebs der Trachea und Bronchien. Virchows Arch path Anat 1871; 53: 470-484

${ }^{6}$ Perls M. Beiträge zur Geschwulstlehre I: Zur Casuistik des Lungenkarzinoms. Virchows Arch path Anat 1872; 56: 437-467

${ }^{7}$ Rindfleisch E. Lehrbuch der Pathologischen Gewebelehre. 3. Auflage. Leipzig: Engelmann, 1873: 403

${ }^{8}$ Wilks S, Moxon W. Lectures on Pathological Anatomy. Sec. Edition. London: Churchill, 1875: 351

9 Pässler H. Über das primäre Karzinom der Lunge. Virchows Arch path Anat 1896; 145: $191-278$

${ }^{10}$ Briese. Zur Kenntnis des primären Lungenkarzinoms, mit statistischen Angaben. Frankf Z Path 1920; 23: 48-55

${ }^{11}$ Wegelin C. Der Bronchial- und Lungenkrebs. Schweiz med Wschr 1942; 72: $1053-1063$

12 Kikuth W. Über Lungenkarzinom. Virchows Arch path Anat 1925; 255 $107-128$

${ }^{13}$ Werner W. Eine Karzinomsektionsstatistik 1920-1951. Arch Geschwforsch 1953; 5: 334-351

${ }^{14}$ Grosse H. Hundert Jahre Lungenkrebs-Statistik des Pathologischen Institutes Dresden-Friedrichstadt. Arch Geschwforsch 1953; 5: $318-334$

${ }^{15}$ WHO-International Agency for Research on Cancer. Cancer Incidence in Five Continents. Vol VI. Lyon, 1992

${ }^{16}$ Jahresbericht Krebsregister Saarland. Morbidität und Mortalität an bösartigen Neubildungen im Saarland. 1996/97

${ }^{17}$ Dhom G. Lungenkrebs im Saarland: Morbidität und Mortalität. Arbeits- und Gesundheitsschutz im Steinkohlenbergbau gestern und heute. A.G. des Saarlandes zur Erforschung und Förderung des Gesundheitsschutzes im Bergbau e.V. (AgiB), 2002: 145-158

${ }^{18}$ Siegmund H. Krebsentwicklung in Bronchiektasen. Virchows Arch path Anat 1922; 236: $191-206$

19 Berblinger W. Die Zunahme des primären Lungenkrebses in den Jahren 1920 - 1924. Klin Wschr 1925; 4: 913 - 916

${ }^{20}$ Fahr Th. Diskussionsbemerkung zu Teutschländer: „Über Metaplasie und Krebsbildungen“. Verh Dtsch Path Ges 1923; 19: 192

${ }^{21}$ Lickint F. Tabak und Tabakrauch als ätiologischer Faktor des Karzinoms. Z Krebsforsch 1930; 30: 349-365

22 Lickint F. Der Bronchialkrebs der Raucher. Münch med Wschr 1935; 82: $1232-1234$

${ }^{23}$ Roffo AH. Der Tabak als krebserzeugendes Agens. Dtsch med Wschr 1937; 63: 1267-1271

${ }^{24}$ Müller FH. Tabakmissbrauch und Lungenkarzinom. Z Krebsforsch 1940; 49: $57-85$

${ }^{25}$ Wynder EL, Graham EA. Tobacco smoking as a possible etiologic factor in bronchogenic carcinoma. J Am Med Ass 1950; 143: 329-336

${ }^{26}$ Druckrey H, Preussmann R. Zur Entstehung carcinogener Nitrosamine am Beispiel des Tabakrauchs. Naturwiss 1962; 49: 498

${ }^{27}$ Härting FA, Hesse W. Der Lungenkrebs, die Bergkrankheit in den Schneeberger Gruben. 4-Jahresschrift Gerichtliche Medizin und Öffentliches Gesundheitswesen 1879; 30/31: 296-309/102-132, $313-337$

${ }^{28}$ Rostoski, Saupe, Schmorl. Die Bergkrankheit der Erzbergleute in Schneeberg in Sachsen („Schneeberger Lungenkrebs“). Z Krebsforsch 1926; 23: 360-384

${ }^{29}$ Piekarski C, Morfeld P. Occupational health aspects of uranium mining in Thuringia and Saxony: An historical view. Appl Occup Environ 1997 12: $915-918$

${ }^{30}$ Kreuzer M, Brachner A, Lehmann F et al. Characteristics of the German uranium miners cohort study. Health Phys 2002; 83: 26-34

${ }^{31}$ Kreuzer M, Müller KM, Brachner A et al. Histopathologic findings of lung carcinoma in German uranium miners. Cancer 2000; 89: $2613-2621$
${ }^{32}$ Morfeld P, Lampert K, Emmerich M et al. Staubexposition, Pneumokoniose und Lungenkrebs: Eine epidemiologische Studie aus dem saarländischen Steinkohlenbergbau. Zbl Arbeitsmed Arbeitsschutz Ergonomie 2002; 52: 382 - 397

${ }^{33}$ Hauptverband Gewerblicher Berufsgenossenschaften. Pers. Mitteilung 2004

${ }^{34}$ Gilson JC. Man and Asbestos Conference New York Academy of Sciences „Biological Effects of Asbestos“. Ann New York Academy of Sciences 1965; 32: 9-11

${ }^{35}$ Nordmann M. Der Berufskrebs der Asbestarbeiter. Z Krebsforsch 1938 47: $288-302$

${ }^{36}$ Jacob G, Anspach M. Pulmonary neoplasia among Dresden asbestos workers. Ann New York Academy of Sciences 1965; 132: 536-548

${ }^{37}$ Selikoff JJ, Hammond EC, Churg J. Asbestos exposure, smoking and neoplasia. J Am Med Assoc 1968; 204: 106 - 112

38 Kreyberg L. Histological lung cancer types. Acta Path Microbiol Scand 1962; Suppl 157

39 Fischer W. Die Gewächse der Lunge und des Brustfells. In: Henke F, Lubarsch $\mathrm{O}$ (Hrsg). Handbuch spezielle pathologische Anatomie und Histologie. III. Band/3. Teil. Berlin: Springer, 1931: 509-606

${ }^{40}$ Kahlau G. Der Lungenkrebs. Erg allg Path path Anat 1957; 37: $258-419$

${ }^{41}$ Lüdecke $\mathrm{H}$. Bronchialkarzinom und Obstruktionspneumonie. Langenbecks Arch Dtsch Z chir 1953; 277: 36-88

42 Auerbach O, Gere B, Forman B et al. Changes in the bronchial epithelium in relation to smoking and cancer of the lung. New Engl J Med 1957; 256: $97-267$

${ }^{43}$ Krompecher E. Basalzellen, Metaplasie und Regeneration. Beitr path Anat 1924; 72: $163-183$

${ }^{44}$ Barnard WG. The nature of the "oat-celled sarcoma" of the mediastinum. J Path Bact 1926; 29: 241 - 244

${ }^{45}$ Marchesani W. Über den primären Bronchialkrebs. Frankf Z Path 1924; 30: $158-190$

${ }^{46}$ Watson WL, Berg JB. Oat cell lung cancer. Cancer 1962; 15: 759- 768

${ }^{47}$ Bensch KG, Corrin B, Pariente R et al. Oat-cell carcinoma of the lung. Cancer 1968; 22: 1163 - 1172

48 Fröhlich F. Die „Helle Zelle“ der Bronchialschleimhaut und ihre Beziehungen zum Problem der Chemorezeptoren. Frankf Z Path 1949; 60: $517-559$

${ }^{49}$ Feyrter F. Über die Argyrophilie des Helle-Zellen-Systems im Bronchialbaum des Menschen. Z mikrosk anat Forsch 1954; 61: 73-81

${ }^{50}$ Cheng CL, Lin TC, Lin TT et al. Cushing's syndrome associated with bronchogenic carcinoma of the lungs. Report of a case with autopsy findings. Chinese Med J 1958; 77: 260-261

${ }^{51}$ Kreyberg L, Liebow AA, Uehlinger E. Histological Typing of Lung Tumours. Genf: WHO, 1977

52 WHO. Histological Classification of Lung Tumours. 1977

53 Malassez L. Examen histologique d'un cas de cancer encéphaloide du poumon (épithéliome). Arch Physiol norm path 1876; 3: 353-372

54 Musser JH. Primary cancer of the lung. Tr A Am Phys 1903; 18: 624-636

${ }^{55}$ Eck H. Über den sog. Alveolarzellkrebs („Lungenadenomatose“). Z Krebsforsch 1955; 60: 433-444

${ }^{56}$ Skorpil F. Beitrag zur Pathologie und Histologie des Alveolarepithelkarzinoms. Frankf Z Path 1941; 55: 347-363

${ }^{57}$ Delarue NC, Graham EA. Alveolar cell carcinoma of the lung (pulmonary adenomatosis, Jagziekte?). J Thorac Surg 1949; 18: 237-251

${ }^{58}$ Fanconi A. Lungenadenomatose. Schweiz med Wschr 1956; 86: $408-411,434-437$

${ }^{59}$ Müller KM. Lungentumoren. In: Doerr W, Uehlinger E (Hrsg). Spezielle Pathologische Anatomie Bd. 16/II. Berlin-Heidelberg-New York-Tokio: Springer, 1983: 1191

60 Watson WL, Smith RR. Terminal bronchiolar or „alveolar cell“ cancer of the lung. J Am Med Assoc 1951; 147: 7-13

${ }^{61}$ Nash G, Langlinais PC, Greenawald KA. Alveolar cell carcinoma: Does it exist? Cancer 1972; 29: $322-326$

62 Greenberg SD, Smith MN, Spjut HJ. Bronchiolo-alveolar carcinomacell of origin. Am J Clin Path 1975; 63: 153 - 167

${ }^{63}$ Schön D, Bertz J, Görsch B et al. Entwicklung der Überlebensraten von Krebspatienten in Deutschland. Berlin: Robert Koch-Institut, 1999 